\title{
ARTICLE OPEN \\ Quantum-optimal detection of one-versus-two incoherent optical sources with arbitrary separation
}

\author{
Xiao-Ming $\mathrm{Lu}^{1,2}$, Hari Krovi ${ }^{3}$, Ranjith $\mathrm{Nair}^{2}$, Saikat Guha ${ }^{4}$ and Jeffrey H. Shapiro (D) $^{5}$
}

We analyze the fundamental quantum limit of the resolution of an optical imaging system from the perspective of the detection problem of deciding whether the optical field in the image plane is generated by one incoherent on-axis source with brightness $\epsilon$ or by two $\epsilon / 2$-brightness incoherent sources that are symmetrically disposed about the optical axis. Using the exact thermal-state model of the field, we derive the quantum Chernoff bound for the detection problem, which specifies the optimum rate of decay of the error probability with increasing number of collected photons that is allowed by quantum mechanics. We then show that recently proposed linear-optic schemes approach the quantum Chernoff bound-the method of binary spatial-mode demultiplexing (B-SPADE) is quantum-optimal for all values of separation, while a method using image inversion interferometry (SLIVER) is near-optimal for sub-Rayleigh separations. We then simplify our model using a low-brightness approximation that is very accurate for optical microscopy and astronomy, derive quantum Chernoff bounds conditional on the number of photons detected, and show the optimality of our schemes in this conditional detection paradigm. For comparison, we analytically demonstrate the superior scaling of the Chernoff bound for our schemes with source separation relative to that of spatially resolved direct imaging. Our schemes have the advantages over the quantum-optimal (Helstrom) measurement in that they do not involve joint measurements over multiple modes, and that they do not require the angular separation for the two-source hypothesis to be given a priori and can offer that information as a bonus in the event of a successful detection.

npj Quantum Information (2018)4:64; https://doi.org/10.1038/s41534-018-0114-y

\section{INTRODUCTION}

The influential Rayleigh criterion for imaging resolution, ${ }^{1}$ which specifies a minimum separation for two incoherent light sources to be distinguishable by a given imaging system, is based on heuristic notions. As pointed out by Feynman [ref. ${ }^{2}$, Sec. 30-4]: "Rayleigh's criterion is a rough idea in the first place..." and a better resolution can be achieved "... if sufficiently careful measurements of the exact intensity distribution over the diffracted image spot can be made..." The fundamental measurement noise is the quantum noise necessarily accompanying any measurement. A more rigorous approach to the resolution measure that accounts for the quantum noise in ideal spatially resolved image-plane photon counting can be formulated using the classical Cramér-Rao bound on the minimum estimation error for locating the sources. ${ }^{3,4}$ Very recently, using methods of quantum estimation theory, ${ }^{5,6}$ it was found that the estimation of the separation between two incoherent sources below the Rayleigh criterion can be drastically improved by measurements employing pre-detection linear-optic processing of the collected light, followed by photon counting. ${ }^{7-20}$

Besides the minimum error of estimating the separation of two point sources, the resolving power of an imaging system can also be studied via the paradigmatic detection problem of deciding whether the optical field in the image plane is generated by one source or two sources. ${ }^{21-25}$ This detection perspective is especially relevant to the detection of binary stars and exoplanets ${ }^{23,26}$ and the detection of protein multimers with fluorescence microscopes. ${ }^{27}$ In a pioneering work, ${ }^{22}$ Helstrom obtained the mathematical description of the quantum-optimal measurement that minimizes the error probability for detecting one or two point sources emitting quasi-monochromatic thermal light. Unfortunately, in addition to having no known physical realization, his method requires the separation between the two hypothetical sources to be given, though this separation is usually unknown in practice.

Here we investigate the performance of two practical quantum measurements for the detection of weak incoherent quasimonochromatic point light sources. We assess the performance of these measurements vis-a-vis direct imaging and the optimum quantum measurement using the asymptotic error exponent (or Chernoff exponent), which specifies the rate at which the error probability decreases exponentially as the observation time or number of received photons increases. We show that a binary spatial-mode demultiplexing (B-SPADE) scheme $^{7}$ is quantumoptimal for all values of separations in the following two senses: (1) the asymptotic error exponent attains the maximum allowed by quantum mechanics, and (2) the error probability of a simple decision rule based on the observations of the B-SPADE is close to the quantum limit. We also show that the scheme of superlocalization by image inversion interferometry (SLIVER) ${ }^{8,10}$ is nearoptimal for sub-Rayleigh separations. The Chernoff exponents of

\footnotetext{
'Department of Physics, Hangzhou Dianzi University, 310018 Hangzhou, China; ${ }^{2}$ Department of Electrical and Computer Engineering, National University of Singapore, 4 Engineering Drive 3, Singapore 117583 , Singapore; ${ }^{3}$ Quantum Information Processing Group, Raytheon BBN Technologies, Cambridge, MA 02138, USA; ${ }^{4}$ College of Optical Sciences, University of Arizona, 1630 E. University Blvd., Tucson, AZ 85719, USA and ${ }^{5}$ Research Laboratory of Electronics, Massachusetts Institute of Technology, Cambridge, MA 02139, USA

Correspondence: X-M. Lu (Ixm@hdu.edu.cn)
}

Received: 16 February 2018 Accepted: 15 November 2018

Published online: 07 December 2018 
both schemes are shown to be superior to that of ideal shot-noiselimited continuum direct imaging in the sub-Rayleigh regime. In addition to their superiority over direct imaging, our methods do not require the capability to perform joint quantum measurements, do not require the two-source separation to be known a priori, can offer an accurate estimate of this separation in the event of a successful detection, ${ }^{7-14}$ and rely on methods that have been experimentally demonstrated in the context of parameter estimation. ${ }^{17-20}$ These advantages over the Helstrom measurement $^{22}$ hold tremendous promise for practical detection applications in both astronomy ${ }^{23}$ and molecular imaging. ${ }^{27}$

\section{RESULTS}

One source versus two sources

The set-up considered in this work is illustrated in Fig. 1. Under hypothesis $\mathrm{H}_{1}$, we have a single thermal source of brightness (average photon number per temporal mode) $\epsilon$ imaged at the origin of the image plane. Under hypothesis $\mathrm{H}_{2}$, we have two thermal sources, each of strength $\epsilon / 2$, located a distance $d$ apart and imaged at the points $\pm \mathbf{d} / 2=( \pm d / 2,0)$ in the image plane. To focus on the resolution power of an optical imaging system, the total brightness is assumed to be identical under the two hypotheses so that simple photon counting is ineffective as a decision strategy. Similarly, the sources are presumed to have identical frequency spectra so that spectroscopy cannot help to distinguish the hypotheses. A strategy for accepting one or the other hypothesis, known as a decision rule, is given by partitioning the space of observations $\mathcal{Z}$ (which is determined by the choice of measurement) into two disjoint regions $\mathcal{Z}_{1}$ and $\mathcal{Z}_{2}$; the onesource hypothesis $H_{1}$ is accepted if the observation belongs to $\mathcal{Z}_{1}$, and $\mathrm{H}_{2}$ is accepted otherwise. The performed quantum measurement can be described by a positive-operator-valued measure (POVM) $\{E(z)\}_{z \in \mathcal{Z}}$, where $z$ denotes the outcome, and the $E(z)$ 's are nonnegative operators resolving the identity operator as $\int \mathrm{d} \mu(z) E(z)=1$ with $\mu(z)$ being an appropriate measure on $\mathcal{Z}^{5,6,28}$ Define $E_{1}=\int_{z \in \mathcal{Z}_{1}} \mathrm{~d} \mu(z) E(z)$ and $E_{2}=\int_{z \in \mathcal{Z}_{2}} \mathrm{~d} \mu(z) E(z)$. Let $\rho_{1}$ and $\rho_{2}$ be the density operators for the fields arriving at the image plane per temporal mode under $\mathrm{H}_{1}$ and $\mathrm{H}_{2}$, respectively. Assuming a flat emission spectrum over the bandwidth $W$, the probabilities of the false-alarm (accepting $\mathrm{H}_{2}$ when $\mathrm{H}_{1}$ is true) and miss (accepting $\mathrm{H}_{1}$ when $\mathrm{H}_{2}$ is true) errors for one-source-versus-two testing are given by $a \equiv \operatorname{Tr}\left(E_{2} \rho_{1}^{\otimes M}\right)$ and $\beta \equiv \operatorname{Tr}\left(E_{1} \rho_{2}^{\otimes M}\right)$, respectively, where $M \simeq W T$ with $T$ being the observation time is the number of available temporal modes (also called the sample size). Assuming prior probabilities $p_{1}$ and $p_{2}$ for the respective hypotheses, the average probability of error is

$P_{\mathrm{e}} \equiv p_{1} a+p_{2} \beta$,

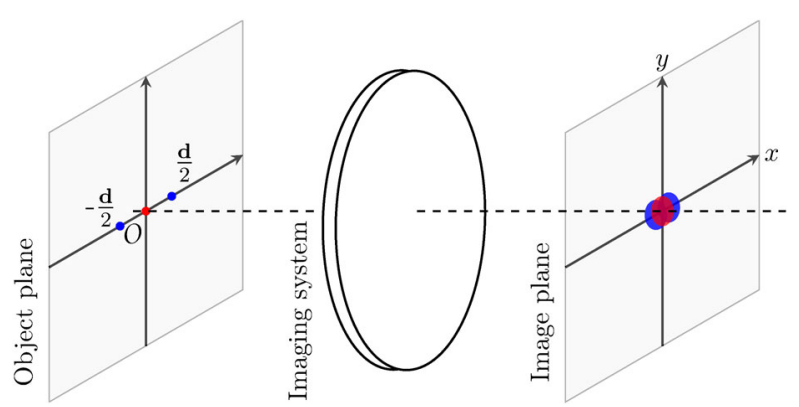

Fig. 1 Schematic of the imaging of incoherent point light sources by a spatially-invariant imaging system. The images of two point sources of equal brightness (blue) spaced closer than the width of the point-spread function (PSF) of the imaging system are difficult to distinguish from that of one point source of the same total brightness (red) located midway between them which is widely used to assess the performance of a quantum decision strategy constituted by a quantum measurement and a classical decision rule. ${ }^{5}$ The minimum error probability optimized over all quantum decision strategies is given by the Helstrom formula ${ }^{5}$

$P_{\mathrm{e}, \min }=\frac{1}{2}\left(1-\left\|p_{2} \rho_{2}^{\otimes M}-p_{1} \rho_{1}^{\otimes M}\right\|_{1}\right)$,

where $\|A\|_{1} \equiv \operatorname{Tr} \sqrt{A^{\dagger} A}$ is the trace norm. The minimum error probability can be achieved by the Helstrom-Holevo test in which $E_{2}$ is taken to be the projector onto the eigen subspace of $p_{2} \rho_{2}^{\otimes M}-p_{1} \rho_{1}^{\otimes M}$ with positive eigenvalues., ${ }^{5,29}$ We refer to this optimal measurement as the Helstrom measurement henceforth.

While the Helstrom formula Eq. (2) allows exact computation of the optimum error probability in principle, it is difficult to physically implement the Helstrom measurement for several reasons. Firstly, the optimal measurement is a joint one over multiple samples. ${ }^{28}$ Secondly, this measurement depends on the separation between the two hypothetic point sources, which is often unknown in the first place. Lastly, the optimal measurement in general depends on the ratio of the prior probabilities of the two hypotheses, whose determination is often subjective. To circumvent these difficulties, we study the performance of two realizable measurements: $\mathrm{B}-\mathrm{SPADE}^{7}$ and $\mathrm{SLIVER}^{8}$ originally introduced in the context of estimating the separation between two closely-spaced incoherent point sources.

\section{B-SPADE}

Spatial-mode demultiplexing refers to spatially separating the image-plane optical field into its components in any chosen set of orthogonal spatial modes. ${ }^{7}$ The binary version of spatial-mode demultiplexing, B-SPADE, uses a device that separates a specific spatial mode from all other modes orthogonal to it, and on-off detectors (that can only distinguish between zero and one or more photons) are placed at the two output ports. In our set-up, the selected spatial mode is chosen to be that generated by the point source at the origin of the object plane. Such a separation of modes is always possible in principle for any given point-spread function (PSF), and various linear-optics schemes can be envisaged to realize it. ${ }^{30-32}$

\section{SLIVER}

The second practical measurement we consider is SLIVER, which separates the optical field at the image plane into its symmetric and antisymmetric components with respect to inversion at the origin, followed by on-off photon detection in the respective ports. ${ }^{8}$ Here, we assume that the PSF is reflection-symmetric in the $y$-axis, i.e., $\psi(-x, y)=\psi(x, y)$, and consider a modified SLIVER for which the inversion operation is replaced by the reflection operation about the $y$-axis-this modification corresponds to the Pix-SLIVER scheme of ref. ${ }^{10}$ with single-pixel (bucket) on-off detectors at the two outputs. For simplicity, we refer to this modified version as SLIVER henceforth. All photodetectors in both B-SPADE and SLIVER are assumed free from dark counts, or at least that the dark-count rate is so far below the signal-count rate as to be negligible.

\section{Asymptotic error (Chernoff) exponents}

In realistic imaging situations, we usually deal with a large sample size $M \gg 1$, which motivates using the asymptotic error exponent as a useful metric for comparing the performance of different measurement schemes against the Helstrom measurement. For any specific quantum measurement performed on each sample, it is known that the minimum error probability $P_{\mathrm{e}, \mathrm{min}}^{\text {(meas) }}$ over all decision rules decreases exponentially in $M$ as 
$P_{\mathrm{e}, \text { min }}^{\text {(meas) }} \sim \exp \left(-M \xi^{\text {(meas) }}\right)$. The asymptotic error exponent $\xi^{\text {(meas) }}=-\lim _{M \rightarrow \infty} \frac{1}{M} \log P_{\text {e.min }}^{\text {(meas) }}$ can be given by the Chernoff exponent (also known as Chernoff information or Chernoff distance), ${ }^{33-35}$ namely,

$\xi^{(\text {meas })}=-\log \min _{0 \leq s \leq 1} \int \mathrm{d} \mu(z) \Lambda_{1}(z)^{s} \Lambda_{2}(z)^{1-s}$,

where $\Lambda_{j}(z)=\operatorname{Tr}\left[E(z) \rho_{j}\right]$ is the probability of obtaining the outcome $z$ under the hypothesis $\mathrm{H}_{j}$, and $\{E(z)\}$ is the POVM for the measurement. On the other hand, the error probability $P_{\mathrm{e}, \mathrm{min}}$ of the optimum quantum measurement (which is in general a joint measurement on the $M$ samples) scales with the exponent $\xi$ known as the quantum Chernoff exponent, which is given by: ${ }^{36-40}$

$\xi \equiv-\log \min _{0 \leq s \leq 1} \operatorname{Tr}\left(\rho_{1}^{s} \rho_{2}^{1-s}\right)$.

Note that $\xi$ is independent of the measurement and $\xi \geq \xi^{\text {(meas) }}$ holds for any measurement.

To calculate the Chernoff exponent, we need to know the characteristics of the imaging system. Without essential loss of generality, we suppose that the imaging system is spatially invariant and of unit magnification ${ }^{41}$ and is described by its 2-D amplitude PSF $\psi(\mathbf{r})$, where $\mathbf{r}=(x, y)$ is the transverse coordinate in the image plane $\mathcal{I}$. We take the PSF to be normalized, i.e., $\int_{\mathcal{I}} \mathrm{d} x \mathrm{~d} y|\psi(x, y)|^{2}=1$. For thermal sources, we show that the exact Chernoff exponents $\xi^{(B-S P A D E)}$ and $\xi^{(\text {SLIVER })}$ for B-SPADE and SLIVER respectively and the quantum Chernoff exponent $\xi$ are given by (see the Methods)

$$
\begin{aligned}
& \xi^{(\mathrm{B}-\mathrm{SPADE})}=\xi=\log \left[\left(1+\epsilon_{-}\right)\left(1+\epsilon_{+}-\mu^{2} \epsilon_{+}\right)\right], \\
& \xi^{(\mathrm{SLIVER})}=\log \left(1+\epsilon_{-}\right) \leq \xi,
\end{aligned}
$$

where the $d$-dependent quantities

$\epsilon_{ \pm}=\frac{1 \pm \delta(d)}{2} \epsilon$ and $\mu=\delta\left(\frac{d}{2}\right) \sqrt{\frac{2}{1+\delta(d)}}$

are defined in terms of the overlap function of the PSF for displacements along the $x$ axis:

$$
\delta(d):=\int_{\mathcal{I}} \mathrm{d} x \mathrm{~d} y \psi^{*}(x, y) \psi(x-d, y) .
$$

Moreover, we here assume that the overlap function (and hence $\epsilon_{ \pm}$and $\mu$ ) is real-valued. This assumption is satisfied for inversionsymmetric PSFs, i.e., $\psi(x, y)=\psi(-x,-y)$, and $y$-axis reflectionsymmetric PSFs, i.e., $\psi(x, y)=\psi(-x, y) .^{10,11}$

It can be seen from Eq. (5) that the Chernoff exponent of the BSPADE is always equal to the quantum Chernoff exponent, meaning that B-SPADE is asymptotically optimal. For SLIVER, the Chernoff exponent is in general not quantum-optimal but is close to quantum-optimal in the sub-Rayleigh regime of small $d$, where $\mu$ is close to unity.

We consider three typical kinds of PSFs, corresponding to Gaussian apertures, rectangular hard apertures, and circular hard apertures. The PSFs can be, respectively, written as

$$
\begin{gathered}
\psi_{\text {gaus }}(x, y)=\frac{1}{\sqrt{2 \pi} \sigma} \exp \left(-\frac{x^{2}+y^{2}}{4 \sigma^{2}}\right), \\
\psi_{\text {rect }}(x, y)=\frac{1}{\pi \sqrt{\sigma_{x} \sigma_{y}}} \operatorname{sinc}\left(x / \sigma_{x}\right) \operatorname{sinc}\left(y / \sigma_{y}\right), \text { and } \\
\psi_{\text {circ }}(x, y)=\frac{1}{2 \sqrt{\pi} \sigma_{c}} \operatorname{jinc}\left(\sqrt{x^{2}+y^{2}} / \sigma_{c}\right),
\end{gathered}
$$

where $\operatorname{sinc}(x) \equiv \sin (x) / x, \operatorname{jinc}(x) \equiv 2 J_{1}(x) / x$, and $J_{1}(x)$ is the Bessel function of the first kind. The "characteristic lengths" $\sigma, \sigma_{x}, \sigma_{y}$, and $\sigma_{c}$ are related to the features of apertures as follows. For a Gaussian aperture, which is commonly assumed in fluorescence microscopy, ${ }^{4}$ we have $\sigma=\lambda / 2 \pi \mathrm{NA}$ with $\lambda$ being the free-space center wavelength and NA the effective numerical aperture of the system. For a $D_{x} \times D_{y}$ rectangular aperture, the characteristic length along the $x$ and $y$ directions are given by $\sigma_{x}=\lambda F / \pi D_{x}$ and $\sigma_{y}=\lambda F / \pi D_{y}$, respectively, where $F$ is the distance between the aperture plane and the image plane in a unit magnification system. For a $D$-diameter circular hard aperture, we have $\sigma_{c}=\lambda F /$ $\pi D$. After some algebra, the overlap functions can be shown to be

$$
\begin{gathered}
\delta_{\text {gaus }}(d)=\exp \left[-d^{2} /\left(8 \sigma^{2}\right)\right], \\
\delta_{\text {rect }}(d)=\operatorname{sinc}\left(d / \sigma_{x}\right), \text { and } \\
\delta_{\text {circ }}(d)=\operatorname{jinc}\left(d / \sigma_{c}\right),
\end{gathered}
$$

using which the Chernoff exponents can be readily obtained.

We plot in Fig. 2 the Chernoff exponents of B-SPADE and SLIVER for the above three PSFs in Eq. (9). We can see that in the subRayleigh regime the Chernoff exponents are insensitive to which PSF is used.
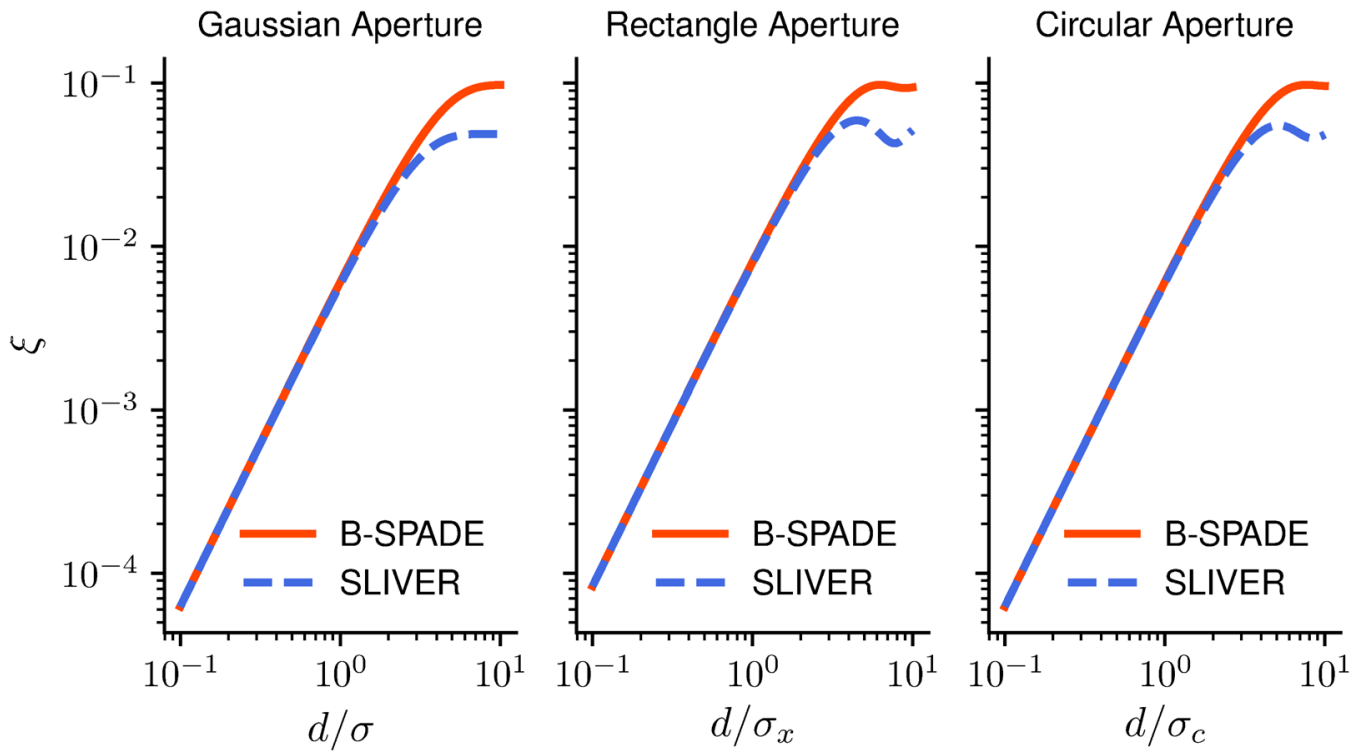

Fig. 2 Chernoff exponents for the B-SPADE and SLIVER measurements as a function of the separation $d$. The quantum Chernoff exponent always equals the Chernoff exponent for the B-SPADE measurement. Here, the total strength $\epsilon$ of the thermal sources is set to be 0.1 
4

Weak-source model

To compare the performance of B-SPADE and SLIVER with that of direct imaging, we introduce the weak-source model. In most applications in optical microscopy and astronomy, the source brightness $\epsilon \ll 1$ photons per temporal mode. ${ }^{7,42-44}$ Then, $\rho_{1}$ and $\rho_{2}$ may be considered with high accuracy to be confined to the subspace consisting of zero or one photons, i.e.,

$\rho_{i} \simeq(1-\epsilon)|\mathrm{vac}\rangle\langle\mathrm{vac}|+\epsilon \eta_{i}$

for $i=1,2$, where $|v a c\rangle$ denotes the vacuum state and $\eta_{i}$ are the corresponding one-photon states obtained by neglecting $O\left(\epsilon^{2}\right)$ terms. This approximation enables us to simplify the theory in comparison with ref. ${ }^{22}$ and still obtain similar results. Moreover, it will enable us to treat the case in which the sources in the twosource hypothesis have different brightnesses while demonstrating the advantages of B-SPADE and SLIVER over direct imaging. Denote the brightnesses of the two point sources under $\mathrm{H}_{2}$ by $\epsilon_{1}$ and $\epsilon_{2}$, satisfying $\epsilon_{1}+\epsilon_{2}=\epsilon$. The one-photon state for two hypothetical sources can be expressed as

$$
\begin{gathered}
\eta(d)=\frac{\epsilon_{1}}{\epsilon}|\Psi(d / 2)\rangle\left\langle\Psi(d / 2)\left|+\frac{\epsilon_{2}}{\epsilon}\right| \Psi(-d / 2)\right\rangle\langle\Psi(-d / 2)| \\
\left|\Psi\left(x^{\prime}\right)\right\rangle \equiv \int_{\mathcal{I}} \mathrm{d} x \mathrm{~d} y \psi\left(x-x^{\prime}, y\right)|x, y\rangle
\end{gathered}
$$

where $|x, y\rangle$ are the one-photon Dirac kets satisfying $\left\langle x, y \mid x^{\prime}, y^{\prime}\right\rangle=$ $\delta\left(x-x^{\prime}\right) \delta\left(y-y^{\prime}\right)$, and $\int_{\mathcal{I}} \mathrm{d} x \mathrm{~d} y|x, y\rangle\langle x, y|=1_{1}$ with $1_{1}$ being the identity operator on the one-photon subspace in the image plane field. ${ }^{7}$ We then have $\eta_{1}=\eta(0)$ and $\eta_{2}=\eta(d)$.

For the specific cases of rectangular and circular hard apertures, Helstrom has derived expressions for the minimum error probability for thermal light sources. ${ }^{22}$ However, these expressions are very complicated. Our weak-source model allows us to simplify the minimum error probability to

$P_{\mathrm{e}, \min }=\sum_{L=0}^{M}\left(\begin{array}{c}M \\ L\end{array}\right)(1-\epsilon)^{M-L} \epsilon^{L} P_{\mathrm{e}, \min \mid L}$,

$P_{\mathrm{e}, \min \mid L} \equiv \frac{1}{2}-\frac{1}{2}\left\|p_{2} \eta_{2}^{\otimes L}-p_{1} \eta_{1}^{\otimes L}\right\|_{1}$.

Here, $\left(\begin{array}{c}M \\ L\end{array}\right)(1-\epsilon)^{M-L} \epsilon^{L}$ is the probability of $L$ photons arriving at the imaging plane and $P_{\mathrm{e}, \min \mid L}$ is the minimum probability of error conditioned on detecting $L$ photons in the image plane. The form of Eq. (13) is due to the fact that the distinguishability between $\rho_{1}$ and $\rho_{2}$ lies in the one-photon sector and the zero-photon event is uninformative. It is implicitly assumed in Eq. (13) that the source flux is low enough that the on-off detectors' recovery time is short compared to the average interarrival time of the photons. Either the conditional error probability of Eq. (14) or the unconditional one of Eq. (13) can be used as a figure of merit, depending on whether or not the number of the photons arriving at the image plane is measured. Helstrom in ref. ${ }^{22}$ took the latter approach, and the performance was studied with respect to the average total number of photons $N=M \epsilon$ detected over the observation interval. On the other hand, in fluorescence microscopy it is common practice to compare the performance of imaging schemes for the same number of detected photons $L^{3,4}$

We can define a conditional Chernoff exponent $\xi_{c}^{\text {(meas) }}$ satisfying $P_{\mathrm{e}, \min \mid L}^{\text {(meas }} \sim \exp \left(-L \xi_{\mathrm{c}}^{\text {(meas) }}\right)$, which is given by Eq. (3) with $\Lambda_{j}(z)$ replaced by the probability of measurement outcomes conditioned on a photon being detected, i.e., $\Lambda_{j}(z)=\operatorname{Tr}\left[E(z) \eta_{j}\right]$. Similarly, the optimum conditional error probability $P_{\mathrm{e}, \min \mid L}$ decays exponentially with $L$ multiplied by the conditional quantum Chernoff exponent given by $\xi_{\mathrm{c}} \equiv-\log \min _{0 \leq s \leq 1} \operatorname{Tr}\left(\eta_{1}^{s} \eta_{2}^{1-s}\right)$. It follows from Eq. (13) that the (unconditional) Chernoff exponents can be obtained via the relation $e^{-\xi}=1-\epsilon+\epsilon e^{-\xi_{c}}$ and $e^{-\xi^{\text {(meas) }}}=1-\epsilon+\epsilon e^{-\xi_{c}^{\text {(meas) }}}$. This implies that the (unconditional)

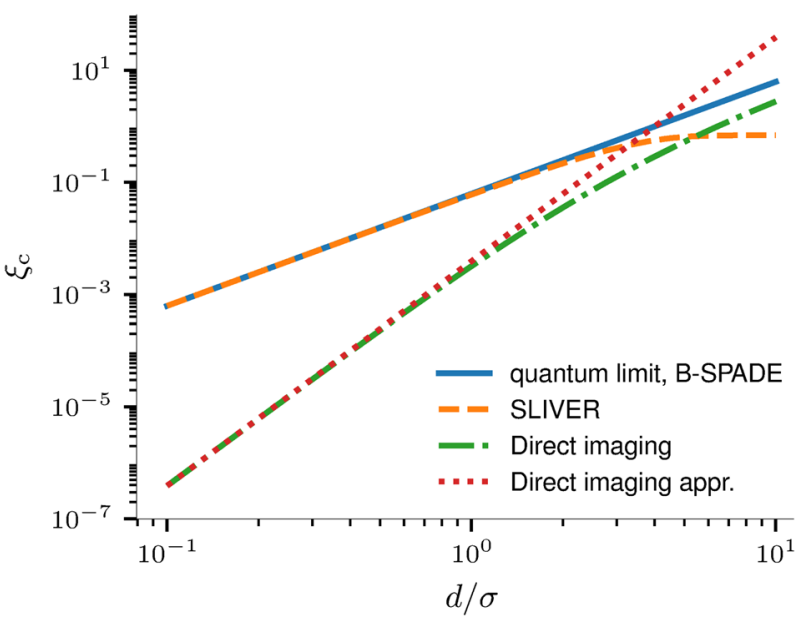

Fig. 3 Conditional Chernoff exponents in the weak-source model as functions of the normalized two-source separation $d$ for the Gaussian PSF of Eq. (9). The Chernoff exponent for B-SPADE (solid) achieves the quantum limit for all values of $d$, while the Chernoff exponent for SLIVER (dashed) is near-quantum-optimal for subRayleigh separations. In this regime, both schemes outperform direct imaging - whose Chernoff exponent is calculated numerically (dash-dotted), as well as by using the small-separation approximation (dotted) given by Eq. (18) - by more than an order of magnitude

Chernoff exponent is monotonically increasing with the conditional one. Particularly, we have $\xi^{\text {meas }} \simeq \varepsilon \xi_{c}^{\text {meas }}$ when $\xi^{\text {meas }} \ll 1$. Therefore, we can use either $\xi^{\text {meas }}$ or $\xi_{c}^{\text {meas }}$ to compare the performance of quantum measurements.

The conditional Chernoff exponents are readily calculated in the weak-source model using Eq. (12):

$\xi_{c}^{(B-S P A D E)}=\xi_{c}=-2 \log \delta(d / 2)$,

$\xi_{\mathrm{c}}^{(\mathrm{SLIVER})}=-\log \frac{1+\delta(d)}{2}$.

Note that as $d$ decreases, the SLIVER result converges to the BSPADE result, which can also be seen in Fig. 3.

The SLIVER measurement is independent of the PSF, while the B-SPADE is adapted according to the PSF. It is remarkable that in the context of estimating the separation of two point optical source, Rehacek et al. $^{13}$ showed there exist complete sets of modes that are optimal for any real symmetric PSF; nevertheless, the PSF-adapted optimal measurement behaves much better than others when fewer modes are measured. In this work, with only two photon detectors, the PSF-adapted B-SPADE is optimal, while the SLIVER is sub-optimal. It remains open whether there exists a complete set of modes that is optimal for the hypothesis-testing scenario considered here, regardless of the details of the PSF. However, similarly to the estimation scenario, the performance of the SLIVER can be definitely enhanced when more modes in the symmetric port are sorted out and measured. This is because the quantum Chernoff exponent is monotonically non-decreasing under quantum operation, ${ }^{40}$ so that sorting out more modes to measure must result a Chernoff exponent not less than that for the SLIVER.

\section{Direct imaging}

Direct imaging (DI) using a charge-coupled device (CCD) camera is a standard detection technique in microscopy and telescopy. ${ }^{4}$ To compare our schemes to direct imaging, we make the conservative assumption of an ideal noiseless photodetector with infinite spatial resolution and unity quantum efficiency placed in the image plane. In the weak-source model, and conditional on a 
photon being detected in a given temporal mode, the observation consists of its position of arrival $(x, y) \in \mathcal{I}$. Using Eq. (12), the resulting probability densities for the observation are $\Lambda_{1}(x, y)=\Upsilon$ $(x, y ; 0)$ and $\Lambda_{2}(x, y)=\Upsilon(x, y ; d)$ under $\mathrm{H}_{1}$ and $\mathrm{H}_{2}$, respectively, where

$\Upsilon(x, y ; d) \equiv \frac{\epsilon_{1}}{\epsilon}|\psi(x-d / 2, y)|^{2}+\frac{\epsilon_{2}}{\epsilon}|\psi(x+d / 2, y)|^{2}$.

We show in the Methods that the conditional Chernoff exponent for ideal DI in the weak-source model scales as $d^{4}$ in the interesting regime of small $d$ :

$\xi_{c}^{(\mathrm{DI})} \simeq \frac{d^{4}}{32} \int_{\mathcal{I}^{\prime}} \mathrm{d} x \mathrm{~d} y \frac{1}{\mathrm{r}(x, y ; 0)}\left[\mathrm{r}^{(2)}(x, y ; 0)\right]^{2}$,

where $\Upsilon^{(n)}(x, y ; d)$ denotes the $n$-th order partial derivative of $\Upsilon(x, y$; d) with respect to $d$, and $\mathcal{I}^{\prime} \equiv\{(x, y) \mid \Upsilon(x, y ; 0)>0\}$. In contrast, the conditional Chernoff exponents of B-SPADE and SLIVER in the weak-source model are of order $d^{2}$, which can be seen by using Eqs. (15) and (16) and $\partial \delta(d) /\left.\partial d\right|_{d=0}=0$.

The conditional Chernoff exponents for different measurements in the case of the Gaussian PSF are given in Table 1. Here, we have used a Taylor series expansion of Eq. (16) in $d$ for SLIVER, and used Eq. (18) for estimating the Chernoff exponent for direct imaging. The characteristic scalings with respect to $d$ of the conditional quantum Chernoff exponent and that of the three measurement schemes are shown in Fig. 3. We see that the Chernoff exponent of SLIVER agrees with the quantum limit for all practical purposes in the sub-Rayleigh regime $d \leq 1$.

\section{Decision rule}

In order to choose a hypothesis based on a sequence of B-SPADE/ SLIVER observations, we need to fix a decision rule. If the separation $d$ is known, the optimal decision rule is given by the likelihood-ratio test: ${ }^{34}$ For a given observation record $\left(z_{1}, z_{2}, \ldots\right.$, $\left.z_{M}\right)$, we choose $\mathrm{H}_{2}$ if $\prod_{j=1}^{M} \Lambda_{2}\left(z_{j}\right) / \Lambda_{1}\left(z_{j}\right)>p_{1} / p_{2}$, where $p_{1}$ and $p_{2}$ are the prior probabilities of $\mathrm{H}_{1}$ and $\mathrm{H}_{2}$, respectively, and choose $\mathrm{H}_{1}$ otherwise. If the separation is unknown, one can use the generalized-likelihood-ratio test, ${ }^{45}$ which first estimates the separation and then does the likelihood-ratio test with the estimated value.

Here, we propose a simplified decision rule that does not require the separation to be known or estimated. Observe that if the detector corresponding to the modes orthogonal to the first mode in the three-mode basis (see Eqs. (23)-(25) in Methods) clicked for any sample, we can infer with certainty (in either source

Table 1. Conditional Chernoff exponents for the Gaussian PSF

\begin{tabular}{ll}
\hline Scheme & Chernoff exponent \\
\hline B-SPADE/Quantum limit & $d^{2} / 16$ \\
SLIVER & $d^{2} / 16-d^{4} / 512+O\left(d^{6}\right)$ \\
Direct imaging & $d^{4} / 256+O\left(d^{6}\right)$ \\
\hline
\end{tabular}

model) that two point sources are present, i.e., $\mathrm{H}_{2}$ is true. The simplified decision rule is then given by accepting $\mathrm{H}_{1}$ only if this detector does not click during the entire observation period.

From Table 2 in the Methods, under the simplified decision rule, the false-alarm probability for $M$ samples is clearly $a=0$ for both the B-SPADE and SLIVER measurement. The miss error probability is the probability that the detector corresponding to $\mathrm{H}_{2}$ does not click, i.e.,

$\beta=\left[\Lambda_{2}\right.$ (off, off $)+\Lambda_{2}($ on, off $\left.)\right]^{M}$,

where $\Lambda_{2}(, ;)$ is the probability of the measurement outcome under $\mathrm{H}_{2}$. It then can shown that $\beta^{\text {(meas) }}=\exp \left(-M \xi^{\text {(meas) }}\right)$ for both the BSPADE and SLIVER measurement.

\section{DISCUSSION}

We have examined the problem of discriminating one thermal source from two closely separated ones for a given diffractionlimited imaging system. Using the exact thermal state of the image-plane field, we have derived the quantum Chernoff exponent for the detection problem. We also have used the weak-source model of the image-plane field, which is very accurate in the optical regime due to the low brightness of a thermal source in each temporal mode, to obtain simple expressions for the Chernoff exponent. The per-sample B-SPADE measurement that separates light in the PSF mode from the rest of the field was shown to attain the quantum-optimal Chernoff exponent for all values of two-source separation. Remarkably, it does so without the need for prior knowledge of the value of $d$, joint measurement over multiple modes, or photon-number resolution in each mode. These properties are not shared by the quantum-optimal measurement elucidated by Helstrom, ${ }^{22}$ which is not a structured receiver. These advantages also adhere to the SLIVER measurement, which is near-quantum-optimal in the subRayleigh regime. Moreover, the experimental design of SLIVER is independent of the particular (reflection-symmetric) PSF of the imaging system.

In fact, the simplified decision rules proposed here for B-SPADE and SLIVER do not require resolving the arrival time of the detected photon or photons. To wit, only a single on-off detector without temporal resolution placed in the output corresponding to the modes orthogonal to the PSF (for B-SPADE) or to the antisymmetric component (for SLIVER) is sufficient for achieving the error probability behavior derived here. Hypothesis $\mathrm{H}_{2}$ is accepted if and only if this detector clicks at any time during the observation period. If we need to simultaneously know the conditional error probability, then at least two photon-numberresolving detectors (or gated on-off detectors with sufficient temporal resolution) are required such that the total number of the photons arriving on the image plane can be obtained from the observation.

Although sophisticated optical microscopy techniques can help resolve multiple sources better than direct imaging, ${ }^{46}$ the

Table 2. Probability distributions of single-sample outcomes for the two on-off detectors used in the B-SPADE and SLIVER measurement

\begin{tabular}{|c|c|c|c|c|c|}
\hline Measurement & Hypothesis & \multicolumn{4}{|c|}{ Probability of event } \\
\hline \multirow[t]{2}{*}{ B-SPADE } & $\mathrm{H}_{1}$ & $\frac{1}{1+\epsilon}$ & $\frac{\epsilon}{1+\epsilon}$ & 0 & 0 \\
\hline & $\mathrm{H}_{2}$ & $\frac{1}{\left(1+\epsilon_{+}\right)\left(1+\epsilon_{-}\right)}$ & $\frac{\mu^{2} \epsilon_{+}}{\left(1+\epsilon_{+}\right)\left(1+\epsilon_{-}\right)\left(1+\epsilon_{+}-\mu^{2} \epsilon_{+}\right)}$ & $\frac{1}{1+\mu^{2} \varepsilon_{+}}-\frac{1}{\left(1+\epsilon_{+}\right)\left(1+\epsilon_{-}\right)}$ & * \\
\hline \multirow[t]{2}{*}{ SLIVER } & $\mathrm{H}_{1}$ & $\frac{1}{1+\epsilon}$ & $\frac{\epsilon}{1+\epsilon}$ & 0 & 0 \\
\hline & $\mathrm{H}_{2}$ & $\frac{1}{\left(1+\epsilon_{+}\right)\left(1+\epsilon_{-}\right)}$ & $\frac{\epsilon_{+}}{\left(1+\epsilon_{+}\right)\left(1+\epsilon_{-}\right)}$ & $\frac{\epsilon_{-}}{\left(1+\epsilon_{+}\right)\left(1+\epsilon_{-}\right)}$ & $\frac{\epsilon_{+} \epsilon_{-}}{\left(1+\epsilon_{+}\right)\left(1+\epsilon_{-}\right)}$ \\
\hline
\end{tabular}


manipulation of the source emission that they require is impossible in astronomical imaging for which the dominant detection technique is direct imaging. Our proof that the linearoptics schemes proposed here can yield Chernoff exponents that are orders of magnitude larger than that of direct imaging, coupled with the rapid recent experimental progress on similar schemes, ${ }^{17-20}$ holds out great promise for applications in astronomy and molecular imaging analysis in the near future.

Some remarks on the assumptions used in this work deserve to be mentioned here. First, the assumption of unit magnification of the imaging system is solely for omitting an irrelevant magnification factor in mathematical expressions and thus does not limit the validity of our result. ${ }^{41}$ Second, we assume the two sources has the same frequency spectra so that we can focus on the resolution power of an imaging system; otherwise, spectroscopy can also be used to distinguish the hypotheses. Third, we assume the brightness of the two sources are identical. For different brightnesses, calculating the quantum Chernoff exponent will become much more difficult, as the symmetric and antisymmetric field are no longer statistically independent, see Methods. However, with the weak-source approximation, we have showed that the Chernoff exponents of the B-SPADE and the SLIVER, as well as their quantum limit, are the same as that for two equal-strength sources, if the optical axis of apparatus is perfectly aligned to the average position of the two point sources. Last, we assume that the optic axis of the devices are perfectly aligned such that the sources are symmetrically located about the optic axis. This may be the biggest potential issue for the practical application of the BSPADE/SLIVER scheme. Some of us have investigated a two-step hybrid scheme, where some of the collected photons are used for the centroid estimation by direct imaging, followed by the BSPADE/SLIVER measurement for the hypothesis testing. A similar scheme was studied for the scenario of estimating the separation of two sources. ${ }^{7,8}$ Such a hybrid configuration is sensible, considered from the following three aspects of direct imaging: (i) it is easy to implement; (ii) it is known to achieve a good centroid estimate; and (iii) it may utilize prior images, e.g., those generated by previous astro-observation. The preliminary simulations, which will be published in elsewhere, show that the hybrid scheme still gives a substantial enhancement for hypothesis testing if half of the photons are employed for centroid estimation and alignment. Besides these assumptions, another possible source of imperfection is dark counts in the photodetectors; this may affect the performance of the B-SPADE and SLIVER schemes, especially those using the simplified decision rules. To improve the robustness against dark counts or extraneous background light, we may use feedback strategies, like those developed in the context of distinguishing between optical coherent states. ${ }^{47,48}$

The quantum Chernoff method used in this work can be possibly extended to multiple sources. For multiple point sources, our method can be generalized in two ways, depending on testing binary or multiple hypotheses. In the first way, we can investigate the resolution power by testing whether there is a weak point light source between two strong point light sources or not. In such a case, the binary hypothesis testing and the Chernoff bound are still applicable. In the second way, we can consider the case where a set of light sources can have several possible configurations and we need to decide which configuration it is. For this problem, we must resort to the multiple Chernoff distance derived in the ref. ${ }^{49}$.

\section{METHODS}

Density operators

To calculate the Chernoff exponent and error probabilities, we need to express the density operators $\rho_{1}$ and $\rho_{2}$ in an appropriate basis. We focus on a single temporal mode $\chi(t)$ of the image-plane field satisfying $\int_{0}^{T}|X(t)|^{2} \mathrm{~d} t=1$ on the observation interval [0, T]. The two mutually incoherent sources at $\pm \mathbf{d} / 2$ under $\mathrm{H}_{2}$ are described by statistically independent zero-mean circular complex Gaussian amplitudes $A_{1}$ and $A_{2}$ with the probability density

$\operatorname{Pr}\left(A_{1}, A_{2}\right)=\prod_{j=1}^{2} \frac{1}{\pi \epsilon_{j}} \exp \left(-\left|A_{j}\right|^{2} / \epsilon_{j}\right)$,

where $\epsilon_{1}$ and $\epsilon_{2}$ are the brightnesses of the two point sources. The imageplane field conditioned on $\left(A_{1}, A_{2}\right)$ is described quantum-mechanically as a coherent-state, i.e., an eigenstate $\left|\phi_{A_{1}, A_{2}}\right\rangle$ of the positive-frequency field operator $\hat{E}^{(+)}(\mathbf{r}, t)$ in the image plane: $\hat{E}^{(+)}(\mathbf{r}, t)\left|\phi_{A_{1}, A_{2}}\right\rangle=\mathcal{E}_{A_{1}, A_{2}}(\mathbf{r}) X(t)\left|\phi_{A_{1}, A_{2}}\right\rangle$, where $\mathcal{E}_{A_{1}, A_{2}}(\mathbf{r})$ is given by

$\mathcal{E}_{A_{1}, A_{2}}(\mathbf{r})=A_{1} \psi(\mathbf{r}-\mathbf{d} / 2)+A_{2} \psi(\mathbf{r}+\mathbf{d} / 2)$

and $\psi(\mathbf{r})$ is the normalized PSF. The density operator under $\mathrm{H}_{2}$ is formally given by

$\rho_{2}=\int_{\mathbb{C}^{2}} d^{2} A_{1} d^{2} A_{2} \operatorname{Pr}\left(A_{1}, A_{2}\right)\left|\phi_{A_{1}, A_{2}}\right\rangle\left\langle\phi_{A_{1}, A_{2}}\right|$.

Note that $\rho_{2}$ depends on the separation $d$ and is reduced to $\rho_{1}$ when setting $d=0$. Moreover, it can be seen from Eq. (21) that under $\mathrm{H}_{1}$ we have $\mathcal{E}_{A_{1}, A_{2}}(\mathbf{r})=\left(A_{1}+A_{2}\right) \psi(\mathbf{r})$. Thus, it is evident that, while the relevant coherent states are defined on a complete set of transverse-spatial modes on $\mathcal{I}$, only three orthonormal modes are in excited (non-vacuum) states. These may be chosen as

$\phi_{1}(\mathbf{r})=\psi(\mathbf{r})$,

$\phi_{2}(\mathbf{r})=\frac{1}{\sqrt{1-\mu^{2}}}\left[\frac{\psi(\mathbf{r}-\mathbf{d} / 2)+\psi(\mathbf{r}+\mathbf{d} / 2)}{2 \sqrt{\lambda_{+}}}-\mu \psi(\mathbf{r})\right]$,

$\phi_{3}(\mathbf{r})=\frac{\psi(\mathbf{r}-\mathbf{d} / 2)-\psi(\mathbf{r}+\mathbf{d} / 2)}{2 \sqrt{\lambda_{-}}}$,

where $\lambda_{ \pm} \equiv(1 \pm \delta(d)) / 2$ and $\mu$ is given by Eq. (7). Using $\left\{\phi_{1}(\mathbf{r}), \phi_{2}(\mathbf{r}), \phi_{3}(\mathbf{r})\right\}$ as a spatial-mode basis, we have

$\mathcal{E}_{A_{1}, A_{2}}(\mathbf{r})=A_{+}\left[\mu \phi_{1}(\mathbf{r})+\sqrt{1-\mu^{2}} \phi_{2}(\mathbf{r})\right]+A_{-} \phi_{3}(\mathbf{r})$,

where $A_{+}=\sqrt{\lambda_{+}}\left(A_{1}+A_{2}\right)$ and $A_{-}=\sqrt{\lambda_{-}}\left(A_{1}-A_{2}\right)$ are two complex random variables. When $\epsilon_{1}=\epsilon_{2}=\epsilon / 2$, the random variables $A_{+}$and $A_{-}$ are statistically independent. ${ }^{8}$ In such a case, we get

$\rho_{1}=\rho_{\text {th }}(\epsilon) \otimes|0\rangle\langle 0|\otimes| 0\rangle\langle 0|$,

$\rho_{2}=U\left[\rho_{\mathrm{th}}\left(\epsilon_{+}\right) \otimes|0\rangle\langle 0|\right] U^{\dagger} \otimes \rho_{\mathrm{th}}\left(\epsilon_{-}\right)$,

where $\rho_{\text {th }}(\epsilon)=\sum_{n}\left[\epsilon^{n} /(\epsilon+1)^{n+1}\right]|n\rangle\langle n|$ is the single-mode thermal state with $\epsilon$ average photons, ${ }^{50,51} U$ is a unitary beamsplitter transformation with transmissivity $\mu$ acting on the first two modes. The $d$-dependent quantities $\epsilon_{ \pm}$and $\mu$ are given by Eq. (7). The transmissivity $\mu$ takes values in the range $[-1,1]$ and equals unit when $d=0$. The beamsplitter implements the transformation

$U|a\rangle \otimes|\beta\rangle=\left|\mu a-\sqrt{1-\mu^{2}} \beta\right\rangle \otimes\left|\mu \beta+\sqrt{1-\mu^{2}} a\right\rangle$

for input coherent states $|\alpha\rangle$ and $|\beta\rangle$, while for a number state-vacuum input $|n\rangle|0\rangle$, we have

$U|n\rangle \otimes|0\rangle=\sum_{k=0}^{n} \sqrt{\left(\begin{array}{l}n \\ k\end{array}\right)} \mu^{k}\left(1-\mu^{2}\right)^{\frac{n-k}{2}}|k, n-k\rangle$.

\section{Quantum Chernoff exponent}

The quantum Chernoff exponent is given by $\xi=-\log \min _{0 \leq s \leq 1} Q_{s}$ with $Q_{s} \equiv \operatorname{Tr}\left(\rho_{1}^{s} \rho_{2}^{1-s}\right)$. Using Eqs. (27), (28) and (30), we get after some algebra:

$$
Q_{s}=\frac{1}{a p^{s}\left(1-b q^{s}\right)},
$$

where the coefficients are

$$
\begin{gathered}
a=\left(1+\epsilon_{+}\right)\left(1+\epsilon_{-}\right), \quad b=\frac{\epsilon_{+}}{1+\epsilon_{+}} \mu^{2}, \\
p=\frac{1+\epsilon}{\left(1+\epsilon_{+}\right)\left(1+\epsilon_{-}\right)}, \quad q=\frac{\epsilon\left(1+\epsilon_{+}\right)}{\epsilon_{+}(1+\epsilon)} .
\end{gathered}
$$

It follows from $0 \leq \epsilon_{ \pm} \leq \epsilon$ and $\epsilon_{+}+\epsilon_{-}=\epsilon$ that $p \leq 1$ and $q \geq 1$. Thus, $Q_{s}$ 
B-SPADE

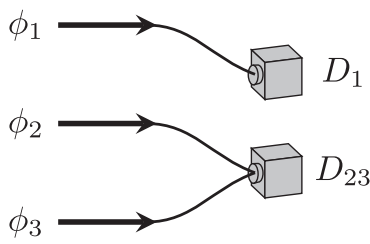

SLIVER

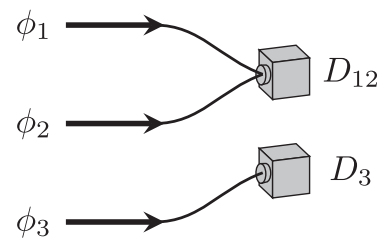

Fig. 4 Effective description of the B-SPADE and SLIVER measurements in the three-mode basis. Here, $\phi_{1}, \phi_{2}$, and $\phi_{3}$ are the three orthonormal modes of Eqs. (23)-(25) used to represent the density operators in Eqs. (27) and (28)

takes its minimum at $s=0$, i.e.,

$\min _{0 \leq s \leq 1} Q_{s}=Q_{0}=\frac{1}{\left(1+\epsilon_{-}\right)\left(1+\epsilon_{+}-\mu^{2} \epsilon_{+}\right)}$,

from which we obtain the quantum Chernoff exponent in Eq. (5).

\section{B-SPADE and SLIVER}

To calculate the Chernoff exponents as well as the error probabilities for BSPADE and SLIVER, it will be convenient to only focus on the effective action of the measurement on the relevant Hilbert subspace. Figure 4 illustrates the effective actions of B-SPADE and SLIVER on the mode subspace spanned by $\phi_{1}(\mathbf{r}), \phi_{2}(\mathbf{r})$, and $\phi_{3}(\mathbf{r})$. The B-SPADE measurement discriminates the first mode from the other two, while the SLIVER measurement discriminates the first two modes from the third, which is the sole excited antisymmetric mode. We emphasize that our schemes are very different from a detector that resolves each of the modes $\phi_{1}(\mathbf{r}), \phi_{2}(\mathbf{r})$, and $\phi_{3}(\mathbf{r})$, since implementing such a detector would require knowledge of $d$. Our schemes, on the other hand, work for any $d$. Using Eqs. (27) and (28) and the effective action of the measurements shown in Fig. 4, the probability distribution of measurement outcomes can be easily obtained as given in Table 2, on which the calculation of the Chernoff exponents is based.

For B-SPADE, we get

$Q_{s}^{(B-S P A D E)}=\frac{1+\tilde{b} \tilde{q}^{s}}{a p^{s}}$,

where $a$ and $p$ are given in Eq. (32), $\tilde{b}=\mu^{2} \epsilon_{+} /\left(1+\epsilon_{+}-\mu^{2} \epsilon_{+}\right)$, and $\tilde{q}=\epsilon\left(1+\epsilon_{+}-\mu^{2} \epsilon_{+}\right) / \mu^{2} \epsilon_{+} \geq 1$. It follows that $Q_{s}$ is minimized over $[0,1]$ by taking $s=0$, leading to the result of $\xi^{(B-S P A D E)}$ in Eq. (5). In the weaksource model, the probability distribution of a detected photon being at the two output ports is $\{1,0\}$ under $\mathrm{H}_{1}$ and $\left\{\delta(d / 2)^{2}, 1-\delta(d / 2)^{2}\right\}$ under $\mathrm{H}_{2}$. Thus, one can easily obtain the result of $\xi_{c}^{(B-S P A D E)}$ as shown in Eq. (15).

For SLIVER, the structure of $\rho_{1}$ and $\rho_{2}$ implies that the two detectors fire independently under both hypotheses. From these equations, the Chernoff exponent of SLIVER can be calculated (and corresponds to $s=0$ as for BSPADE) with the result of Eq. (6). In the weak-source model, the probability distribution of a detected photon being at the two output ports is $\{1,0\}$ under $\mathrm{H}_{1}$ and $\{[\delta(d)+1] / 2,[1-\delta(d)] / 2\}$ under $\mathrm{H}_{2}$. Thus, one can easily obtain the result of $\xi_{c}^{\text {(SLIVER) }}$ as shown in Eq. (16).

\section{Leading term of Chernoff exponent}

For a given measurement scheme, let $\Upsilon(z ; d)$ be the resulting probability density of a measurement outcome $z$, where $d$ is the distance between the two hypothetic point sources. The Chernoff exponent of Eq. (3) for testing $\mathrm{H}_{2}(d>0)$ against $\mathrm{H}_{1}(d=0)$ can be written as $\xi(d)=-\log \min _{0 \leq s \leq 1} Q_{s}(d)$ with $Q_{s}(d) \equiv \int \mathrm{d} \mu(z) \Upsilon(z ; 0)^{1-s} \Upsilon(z ; d)^{s}$. Let us now focus on the leading term of $\xi(d)$ for small separations $d \approx 0$, where the optimal measurement performs much better than direct imaging. We expand $Q_{s}(d)$ in a Taylor series as

$Q_{s}(d)=1+\sum_{k=1}^{\infty} \frac{d^{k}}{k !} Q_{s, k}$,

$\left.Q_{s, k} \equiv \int_{\mathcal{I}^{\prime}} \mathrm{d} \mu(z) \Upsilon(z ; 0)^{1-s} \frac{\partial^{k} \Upsilon(z ; d)^{s}}{\partial d^{k}}\right|_{d=0}$,

where $\mathcal{I}^{\prime} \equiv\{z \mid \mathrm{Y}(z ; 0)>0\}$. These coefficients $Q_{s, k}$ are independent of $d$. Although in our model the separation $d$ is nonnegative, the PSFs in Eq. (9) can be easily extended to real numbers and meanwhile assured to be smooth at $d=0$. It then follows from Eq. (12) that $\Upsilon(z ; d)=\Upsilon(z ;-d)$ and thus all odd derivatives of $\Upsilon(z ; d)$ with respect to $d$ at $d=0$ vanish for an arbitrary $z$. As a result, we have

$$
\begin{gathered}
Q_{s, 1}=Q_{s, 3}=0, \quad Q_{s, 2}=s g^{(2)}(0), \\
Q_{s, 4}=3 s(s-1) \mathcal{K}+s g^{(4)}(0),
\end{gathered}
$$

where $g^{(k)}$ denotes the $k$-th derivatives of $g(d) \equiv \int_{\mathcal{I}^{\prime}} \mathrm{d} \mu(z) \Upsilon(z ; d)$ and

$\mathcal{K} \equiv \int_{\mathcal{I}^{\prime}} \mathrm{d} \mu(z) \frac{1}{\Upsilon(z ; 0)}\left[\frac{\partial^{2} \Upsilon(z ; d)}{\partial d^{2}}\right]_{d=0}^{2}$.

For the ideal direct imaging scenario in the weak-source model, the measurement outcome $z$ is the coordinates of a detected photon in the image plane, i.e., $z=(x, y) \in \mathcal{I}$. Suppose that the two point sources are aligned along the $x$ axis, $\Upsilon(z ; d)$ is then given by Eq. (17) with $z=(x, y)$. For all three typical kinds of PSFs considered in this work, we have $g(d)=1$ for direct imaging. In such a case, the leading $d$-dependent term in the Taylor series of $Q_{s}(d)$ is of fourth order. It then follows that $\xi_{c}^{(\mathrm{DI})} \simeq \frac{d^{4}}{32} \mathcal{K}$, which is Eq. (18). On the other hand, for B-SPADE and SLIVER, it can be seen from Table 2 that $g(d)=\Lambda_{2}$ (off, off) $+\Lambda_{2}$ (on, off), so that $g^{(2)}(0)$ is nonzero and the leading term in $Q_{s}(d)$ is second-order in $d$.

\section{DATA AVAILABILITY}

Additinal data and analysis files are available from the authors on request.

\section{ACKNOWLEDGEMENTS}

We thank Mankei Tsang for several useful discussions. This work was supported by the Zhejiang Provincial Natural Science Foundation of China under Grant No. LY18A050003, the National Natural Science Foundation of China under Grant Nos. 61871162 and 11805048, the Singapore National Research Foundation under NRF Grant No. NRF-NRFF2011-07, the Singapore Ministry of Education Academic Research Fund Tier 1 Project R-263-000-C06-112, the Defense Advanced Research Projects Agency's (DARPA) Information in a Photon (InPho) program under Contract No. HR0011-10-C-0159, the REVEAL and EXTREME Imaging program, and the Air Force Office of Scientific Research under Grant No. FA9550-14-1-0052.

\section{AUTHOR CONTRIBUTIONS}

X.-M.L. performed the weak-source model analysis. H.K., S.G., J.H.S., and R.N performed the thermal-state analysis. R.N. performed the SLIVER analyses. X.-M.L. and R.N. wrote the manuscript. All the authors discussed extensively during the course of this work. This paper extends and unifies preliminary work in the preprints $^{52}$ (thermal-state model) and ${ }^{53}$ (weak-source model).

\section{ADDITIONAL INFORMATION}

Competing interests: The authors declare no competing interests.

Publisher's note: Springer Nature remains neutral with regard to jurisdictional claims in published maps and institutional affiliations.

\section{REFERENCES}

1. Lord Rayleigh, F. R. S. Xxxi. investigations in optics, with special reference to the spectroscope. Philos. Mag. Ser. 5 8, 261-274 (1879).

2. Feynman, R., Leighton, R. \& Sands, M. The Feynman Lectures on Physics:Volume I (Addison-Wesley, Reading, MA, 1963).

3. Ram, S., Ward, E. S. \& Ober, R. J. Beyond Rayleigh's criterion: a resolution measure with application to single-molecule microscopy. Proc. Natl Acad. Sci. USA 103 4457-4462 (2006).

4. Chao, J., Ward, E. S. \& Ober, R. J. Fisher information theory for parameter estimation in single molecule microscopy: tutorial. J. Opt. Soc. Am. A 33, B36-B57 (2016).

5. Helstrom, C. W. Quantum Detection and Estimation Theory (Academic Press, New York, 1976).

6. Holevo, A. S. Probabilistic and Statistical Aspects of Quantum Theory (North-Holland, Amsterdam, 1982).

7. Tsang, M., Nair, R. \& Lu, X.-M. Quantum theory of superresolution for two incoherent optical point sources. Phys. Rev. X 6, 031033 (2016). 
8. Nair, R. \& Tsang, M. Interferometric superlocalization of two incoherent optical point sources. Opt. Express 24, 3684-3701 (2016).

9. Tsang, M., Nair, R. \& Lu, X.-M. Quantum information for semiclassical optics. In Proc. SPIE; Quantum and Nonlinear Optics IV (eds Gong, Q., Guo, G. \& Ham, B.) Vol. 10029, 1002903 (2016).

10. Nair, R. \& Tsang, M. Far-field superresolution of thermal electromagnetic sources at the quantum limit. Phys. Rev. Lett. 117, 190801 (2016).

11. Ang, S. Z., Nair, R. \& Tsang, M. Quantum limit for two-dimensional resolution of two incoherent optical point sources. Phys. Rev. A. 95, 063847 (2017).

12. Lupo, C. \& Pirandola, S. Ultimate precision bound of quantum and subwavelength imaging. Phys. Rev. Lett. 117, 190802 (2016).

13. Rehacek, J., Paúr, M., Stoklasa, B., Hradil, Z. \& Sánchez-Soto, L. L. Optimal measurements for resolution beyond the Rayleigh limit. Opt. Lett. 42, 231-234 (2017).

14. Tsang, M. Subdiffraction incoherent optical imaging via spatial-mode demultiplexing. New J. Phys. 19, 023054 (2017)

15. Kerviche, R., Guha, S. \& Ashok, A. Fundamental limit of resolving two point sources limited by an arbitrary point spread function. In 2017 IEEE International Symposium on Information Theory (ISIT) 441-445 (2017).

16. Yang, F., Nair, R., Tsang, M., Simon, C. \& Lvovsky, A. I. Fisher information for farfield linear optical superresolution via homodyne or heterodyne detection in a higher-order local oscillator mode. Phys. Rev. A 96, 063829 (2017).

17. Tang, Z. S., Durak, K. \& Ling, A. Fault-tolerant and finite-error localization for point emitters within the diffraction limit. Opt. Express 24, 22004-22012 (2016).

18. Yang, F., Taschilina, A., Moiseev, E. S., Simon, C. \& Lvovsky, A. I. Far-field linear optical superresolution via heterodyne detection in a higher-order local oscillator mode. Optica 3, 1148-1152 (2016).

19. Tham, W.-K., Ferretti, H. \& Steinberg, A. M. Beating Rayleigh's curse by imaging using phase information. Phys. Rev. Lett. 118, 070801 (2017).

20. Paúr, M., Stoklasa, B., Hradil, Z., Sánchez-Soto, L. L. \& Rehacek, J. Achieving the ultimate optical resolution. Optica 3, 1144-1147 (2016)

21. Harris, J. L. Resolving power and decision theory. J. Opt. Soc. Am. 54, 606-611 (1964).

22. Helstrom, C. Resolution of point sources of light as analyzed by quantum detection theory. IEEE Trans. Inform. Theory 19, 389-398 (1973).

23. Acuna, C. O. \& Horowitz, J. A statistical approach to the resolution of point sources. J. Appl. Stat. 24, 421-436 (1997).

24. Shahram, M. \& Milanfar, P. Statistical and information-theoretic analysis of resolution in imaging. IEEE Trans. Inform. Theor. 52, 3411-3437 (2006).

25. Dutton, Z., Shapiro, J. H. \& Guha, S. Ladar resolution improvement using receivers enhanced with squeezed-vacuum injection and phase-sensitive amplification. J. Opt. Soc. Am. B 27, A63-A72 (2010).

26. Labeyrie, A., Lipson, S. G. \& Nisenson, P. An Introduction to Optical Stellar Interferometry (Cambridge University Press, Cambridge, 2006).

27. Nan, X. et al. Single-molecule superresolution imaging allows quantitative analysis of raf multimer formation and signaling. Proc. Natl Acad. Sci. USA 110, 18519-18524 (2013).

28. Hayashi, M. Quantum Information: An Introduction 1 edn (Springer-Verlag, Berlin/ Heidelberg, 2006).

29. Holevo, A. Statistical decision theory for quantum systems. J. Multivar. Anal. 3, 337-394 (1973)

30. Reck, M., Zeilinger, A., Bernstein, H. J. \& Bertani, P. Experimental realization of any discrete unitary operator. Phys. Rev. Lett. 73, 58-61 (1994).

31. Morizur, J.-F. et al. Programmable unitary spatial mode manipulation. J. Opt. Soc. Am. A 27, 2524-2531 (2010).

32. Miller, D. A. B. Reconfigurable add-drop multiplexer for spatial modes. Opt. Express 21, 20220-20229 (2013).
33. Chernoff, H. A measure of asymptotic efficiency for tests of a hypothesis based on the sum of observations. Ann. Math. Stat. 23, 493-507 (1952).

34. Van Trees, H. L., Bell, K. L. \& Tian, Z. Detection, Estimation, and Modulation Theory, Part I 2nd edn (Wiley, New York, NY, 2013).

35. Cover, T. \& Thomas, J. Elements of Information Theory 2nd edn (Wiley, New York, NY, 2006).

36. Ogawa, T. \& Hayashi, M. On error exponents in quantum hypothesis testing. IEEE Trans. Inf. Theory 50, 1368-1372 (2004).

37. Kargin, V. On the Chernoff bound for efficiency of quantum hypothesis testing. Ann. Stat. 33, 959-976 (2005).

38. Audenaert, K. M. R. et al. Discriminating states: the quantum Chernoff bound. Phys. Rev. Lett. 98, 160501 (2007).

39. Nussbaum, M. \& Szkoła, A. The Chernoff lower bound for symmetric quantum hypothesis testing. Ann. Stat. 37, 1040-1057 (2009).

40. Audenaert, K., Nussbaum, M., Szkoła, A. \& Verstraete, F. Asymptotic error rates in quantum hypothesis testing. Commun. Math. Phys. 279, 251-283 (2008).

41. Goodman, J. W. Introduction to Fourier Optics 3rd edn (Roberts and Company Publishers, Englewood, CO, 2005).

42. Goodman, J. W. Statistical Optics (John Wiley \& Sons, New York, NY, 1985).

43. Gottesman, D., Jennewein, T. \& Croke, S. Longer-baseline telescopes using quantum repeaters. Phys. Rev. Lett. 109, 070503 (2012).

44. Tsang, M. Quantum nonlocality in weak-thermal-light interferometry. Phys. Rev. Lett. 107, 270402 (2011).

45. Kay, S. M. Fundamentals of Statistical Signal Processing, Volume II: Detection Theory 1st edn (Prentice Hall, Upper Saddle River, NJ, 1998).

46. Weisenburger, S. \& Sandoghdar, V. Light microscopy: an ongoing contemporary revolution. Contemp. Phys. 56, 123-143 (2015).

47. Dolinar, S. J. An optimum receiver for the binary coherent state quantum channel. MIT Res. Lab. Electron. Quart. Progr. Rep. 111, 115-120 (1973).

48. Geremia, J. Distinguishing between optical coherent states with imperfect detection. Phys. Rev. A 70, 062303 (2004).

49. Li, K. Discriminating quantum states: the multiple Chernoff distance. Ann. Stat. 44, 1661-1679 (2016)

50. Mandel, L. \& Wolf, E. Optical Coherence and Quantum Optics (Cambridge University Press, Cambridge, 1995).

51. Shapiro, J. H. The quantum theory of optical communications. IEEE J. Sel. Top. Quant. Elect. 15, 1547-1569 (2009).

52. Krovi, H., Guha, S. \& Shapiro, J. H. Attaining the quantum limit of passive imaging. Preprint at arXiv:1609.00684 (2016).

53. Lu, X.-M., Nair, R. \& Tsang, M. Quantum-optimal detection of one-versus-two incoherent sources with arbitrary separation. Preprint at arXiv:1609.03025 (2016).

(i) Open Access This article is licensed under a Creative Commons adaptation, distribution and reproduction in any medium or format, as long as you give appropriate credit to the original author(s) and the source, provide a link to the Creative Commons license, and indicate if changes were made. The images or other third party material in this article are included in the article's Creative Commons license, unless indicated otherwise in a credit line to the material. If material is not included in the article's Creative Commons license and your intended use is not permitted by statutory regulation or exceeds the permitted use, you will need to obtain permission directly from the copyright holder. To view a copy of this license, visit http://creativecommons. org/licenses/by/4.0/.

(c) The Author(s) 2018 